\title{
Swiss Science Concentrates \\ A CHIMIA Column \\ Short Abstracts of Interesting Recent Publications of Swiss Origin
}

\section{$\mathrm{N}$-Heterocyclic Carbene Coordination to Surface Copper Sites in Selective Semihydrogenation Catalysts from Solid-State NMR Spectroscopy}

N. Kaeffer,* D. Mance, * and C. Copéret* Angew. Chem. Int. Ed. 2020, 59, 19999-20007.

ETH Zurich

Supported metal nanoparticles constitute a very large class of heterogeneous catalysts with multiple applications that include hydrogenation. Improving such types of catalysts requires an understanding of the interactions between the metal surfaces and ligands/substrates at the molecular level, which is often difficult to access. In this work, Kaeffer et al. compared the properties of NHC $(\mathrm{NHC}=N$-heterocyclic carbene) coordinated to silicasupported $\mathrm{Cu}$ single sites and nanoparticles. By combining solidstate NMR spectroscopy and DFT calculations, they demonstrate that the active species in the semihydrogenation of alkynes are supported $\mathrm{Cu}$ nanoparticles, wherein NHC coordination is favoured at $\mathrm{Cu}$ atoms atop of the surface, rather than $\mathrm{Cu}$ single sites linearly sandwiched between the NHC and an oxygen atom of the silica support. This study contributes to bridging the gap between surface science and heterogeneous catalysis.

\section{Authors' comments:}

"With the help of solid-state NMR spectroscopy including DNPSENS, we were delighted to capture a minute molecular interaction - a residual dipolar coupling arising from the quadrupolar nature of the $\mathrm{Cu}$ atom - that in turn enables addressing the precise molecular structure of materials relevant to catalysis."

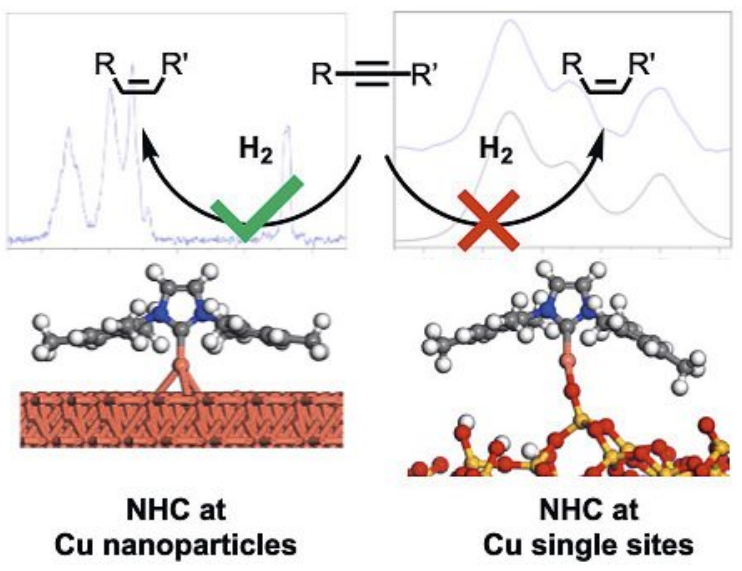

\section{Activation of Copper Species on Carbon Nitride for Enhanced Activity in the Arylation of Amines}

E. Vorobyeva, V. C. Gerken, S. Mitchell,* A. Sabadell-Rendón, R. Hauert, S. Xi, A. Borgna, D. Klose, S. M. Collins, P. A. Midgley, D. M. Kepaptsoglou, Q. M. Ramasse, A. Ruiz-Ferrando, E. Fako, M. A. Ortuño, N. López,* E. M. Carreira,* and J. PérezRamírez.* ACS Catal. 2020, 10, 11069-11080.

ETH Zurich, Institute of Chemical Research of Catalonia, Empa, A*STAR, University of Cambridge, University of Leeds, University of York

The $\mathrm{Cu}$-catalyzed $\mathrm{C}-\mathrm{N}$ cross-coupling of aryl halides has attracted much interest due to the higher sustainability of copper vs noble metals. Cooperative $\mathrm{Cu}-\mathrm{Fe}$ catalytic systems have shown improved performance but the role of $\mathrm{Fe}$ and its synergistic effects with $\mathrm{Cu}$ remains unclear. In this collaborative work, the authors report the promoting effect of graphitic carbon nitride $(\mathrm{GCN})$ in $\mathrm{Cu}$-catalyzed $\mathrm{N}$-arylation. They first prepared $\mathrm{Cu}, \mathrm{Fe}$ and $\mathrm{Cu}-\mathrm{Fe}$ single-atom heterogeneous catalysts (SAHCs) based on GCN derived from dicyandiamide. Whereas these SAHCs alone were inactive in $\mathrm{N}$-arylation, the addition of $\mathrm{Fe}_{1} / \mathrm{GCN}$ to the CuI-catalyzed reaction afforded a fivefold increase in the turnover frequency compared to the standard CuI catalyst. In-depth experimental and computational studies indicated that doping the lattice of GCN with Fe plays a significant role in maximizing the surface confinement of the adsorbed $\mathrm{Cu}$ catalyst. This work advances the design of more efficient heterogeneous catalysts for cross-coupling reactions.

\section{Authors' comments:}

"Based on a cooperative multidisciplinary effort, our study highlights the opportunities and complexity of using metal-coordinating host materials as promoters in coupling chemistry involving soluble catalysts and additives."

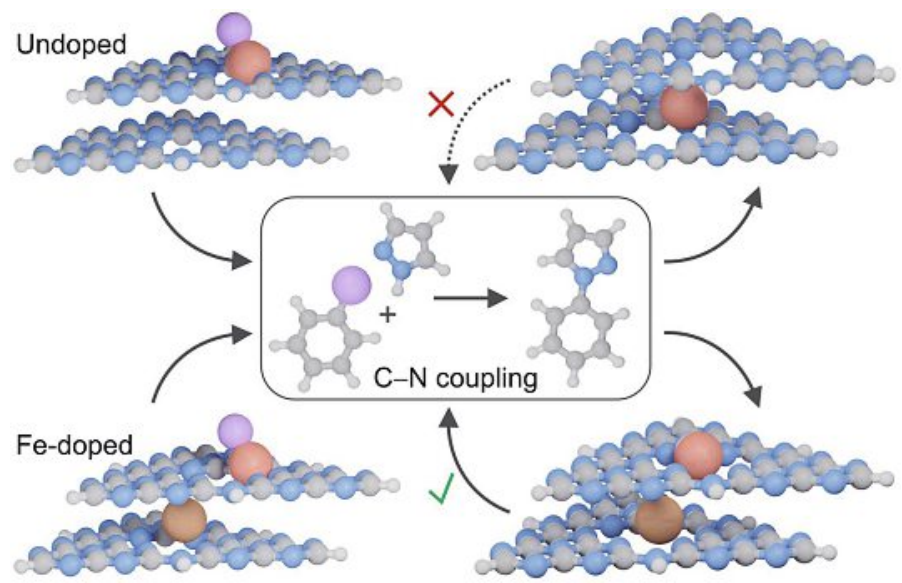




\section{Transfer Learning Enables the Molecular Transformer to Predict Regio- and Stereoselective Reactions on Carbohydrates}

G. Pesciullesi, P. Schwaller T. Laino, and J.-L. Reymond,* Nat. Commun. 2020, 11, 4874.

University of Bern, IBM Research-Europe

Machine learning is a powerful tool in chemical research, which has been applied to structure-activity relationship predictions, virtual screenings and quantum chemistry, among others. In recent years, reaction prediction methods have emerged as important tools for the pharmaceutical industry to optimize chemists' productivity and reduce the number of experiments to perform. In this work, the authors used a set of $20 \mathrm{k}$ carbohydrate reactions from literature including protection/deprotection and glycosylation sequences for transfer learning. They demonstrate that the adapted model, called the Carbohydrate Transformer, performs significantly better than currently used models to predict regioand stereoselectivity. The predictions were validated experimentally via the 14-step synthesis of a lipid-linked oligosaccharide. Overall, a prediction accuracy above $70 \%$ was observed for the current model. This transfer learning approach should be applicable to other challenging reaction classes.

\section{Authors' comments:}

"A very productive collaboration between Machine Learning experts and Organic Chemists. We explored strategies to build 'ad-hoc' reaction prediction models that can be applied to any reaction class. This type of collaborations is beneficial for both fields, we are thrilled to see what comes next"

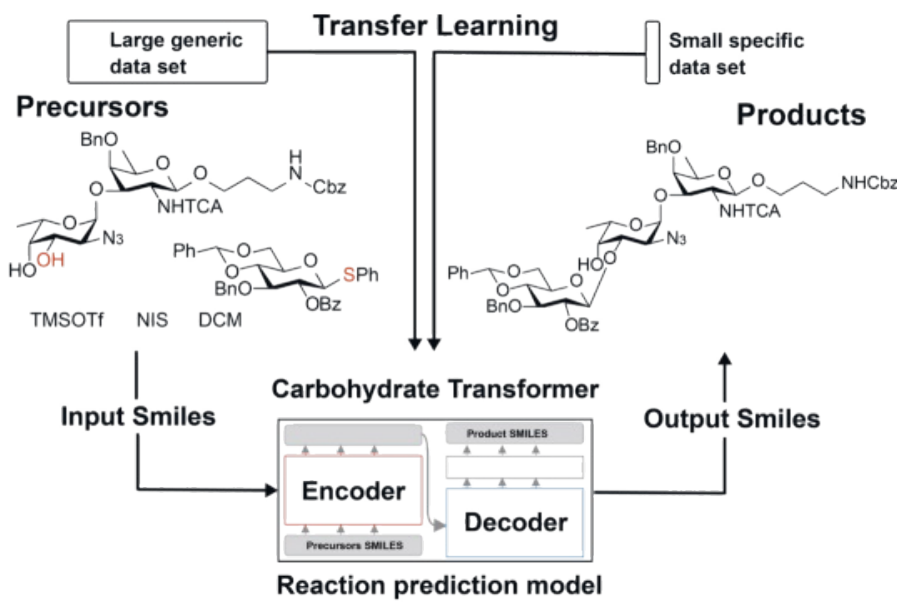

\section{Catalyst-Controlled Transannular Polyketide Cyclization Cascades: Selective Folding of Macrocyclic Polyketides}

F. C. Raps, V. C. Fäseke, D. Häussinger, and C. Sparr,* Angew. Chem. Int. Ed. 2020, 59, 18390-18394.

University of Basel

Aromatic polyketides are a class of natural products with important applications in medicine. Their biosynthesis involves the selective cyclization of linear polyketide chains, which is difficult to reproduce by human hand. In this paper, the authors report catalyst-controlled transannular cyclization cascades allowing to selectively form such polyketide products. Macrocyclic polyketides possessing increased stability and reduced conformational freedom were divergently transformed into aromatic products using different small-molecule catalysts. In particular, aromatic polyketides possessing a fungal or bacterial folding pattern were generated through an aldol/retro-Claisen/aldol condensation cascade. On the other hand, naphthoquinone natural products were synthesized from a partially reduced substrate in the presence of a thiourea catalyst. This strategy advances the biomimetic synthesis of aromatic polyketides and provides an alternative to combinatorial polyketide biochemistry.

\section{Authors' comments:}

"The higher stability of macrocyclic polyketide substrates, compared to their native, linear congeners, enables the catalyst-controlled folding for divergent polyketide cyclizations. Ongoing studies focus on larger macrocycles and increased complexity."

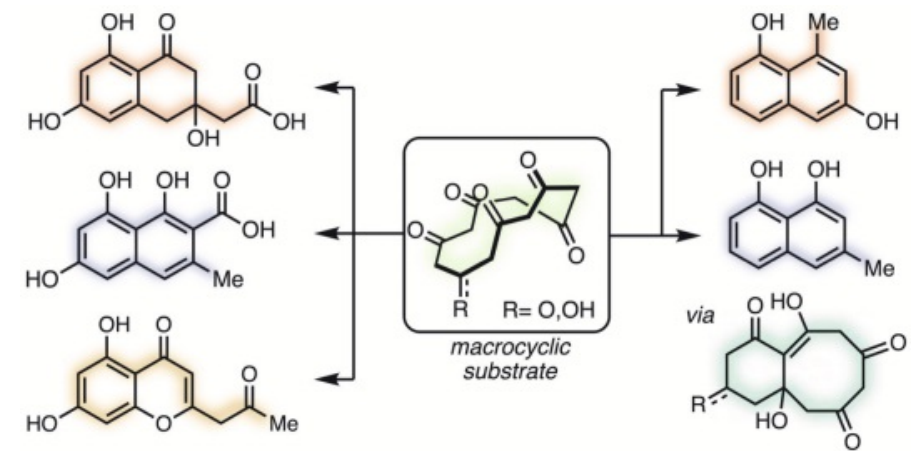

